\title{
Photoactive thin films of terphenylene-based amorphous polymers. Synthesis, electrooptical properties, and role of photoquenching and inner filter effects in the chemosensing of nitroaromatics
}

\author{
Raúl O. Garay*, Pablo G. Del Rosso, Maria J. Romagnoli, Marcela F. Almassio, Ana Belén Schvval \\ INQUISUR, Departamento de Química, CONICET, Universidad Nacional del Sur, Alem 1253, CP 8000, Bahía Blanca, Argentina
}

\section{A R T I C L E I N F O}

\section{Keywords:}

Film chemosensor

Terphenylene choromophores

Fluorescence quenching

Nitroaromatic compounds

Aqueous phase

Segmented conjugated polymer

\begin{abstract}
A B S T R A C T
New photoactive segmented conjugated polymers with terphenylene chromophores were synthesized, and the chemosensing abilities to detect nitroaromatics compounds (NACs) of the polymeric thin films were evaluated in aqueous media. The thin films are strongly fluorescent, amorphous with no aggregation of the chromophores in the solid state and sensitive towards NACs in water. Though true quenching occurring after diffusion of the NACs into the amorphous films contributes to the total response of these polymer films, quenching efficiencies of nitroaromatics are strongly influenced by additional inner filter effect contributions that could be used to increase the film response. Thus, to maximize the response of these polymers, it is convenient to use the shortest feasible $\lambda_{\text {exc }}$ for trinitritoluene (TNT) and the longest feasible $\lambda_{\operatorname{exc}}$ for picric acid (PA). In the micromolar concentration region, the highly absorbing PA frequently has a stronger response than TNT due to the inner filter effects (IFE) contributions. However, we observed that the properties of the material, such as exciton mobility or quencher-polymer compatibilities, become more relevant to define the quenching efficiency at the nanomolar range; though the electrondonor capabilities of the chromophores have no bearing on quenching efficiency. So, the tuning of morphology and photophysical properties of the polymer by structural design should be complemented with a rational selection of experimental conditions, e.g., $\lambda_{\text {exc }}$, in order to enhance the response towards the NAC of interest.
\end{abstract}

\section{Introduction}

Human activity produces significant amounts of waste that often end in water bodies of industrial and city areas [1]. Many contaminants are, with time, mineralized to carbon dioxide and other unharmful compounds. However, certain organic water pollutants, like nitroaromatic compounds (NACs), and in particular, di- and trinitrotoluene are persistent because they are highly oxidized. Even worse, their partial degradation frequently leads to aromatic amines [2,3], which are of higher toxicity than the original pollutant. Fluorescence quenching has fulfilled a notable role in the efforts to develop chemosensors for the detection of NACs [4]. Thus, photoactive films of conjugated polymers have been very successful in the detection of TNT on air above buried explosives [5]. On the other hand, detection of NACs in water media has been demanding because of the hydrophobic nature of fluorescent organic materials [6]. Hence, efforts have been aimed to produce aqueous probes such as water-stable polymeric nanoaggregates [7-10] and monomeric [11-14] or polymeric [15,16] water-soluble organic chromophores. Other sensing configurations tested for detection and quantification of NACs in water are monolayers covalently linked to the substrate [17] and thin films of monomeric or polymeric materials deposited onto rigid substrates [18] or adsorbed on paper [19].

In our view, hydrophobic photoative polymeric films have distinct advantages for their incorporation in portable sensing devices of NACs in aqueous media such as ease of processability, fabrication, storage, and transportation as well as thermal and chemical stability and recyclability [18]. A caveat in film sensing is that both surface area and the amount chromophores available for quenching are reduced compared to nanoaggregates or soluble probes. It is necessary, therefore, that analytes diffuse into the film for achieving better quenching efficiencies; in that way, morphology issues become important in the considerations of material requirements since diffusion depends on both morphology and polymer-analyte interactions. A positive aspect is that the chromophoric array into the film could have amplified responses if tridimensional migration of excitons among chromophores takes place by homo resonance energy transfer (RET) [20-22]. It follows that the increased number of exciton-quencher encounters produced by migration will enhance the quenching efficiency since the

\footnotetext{
* Corresponding author.

E-mail address: rgaray@criba.edu.ar (R.O. Garay).
} 


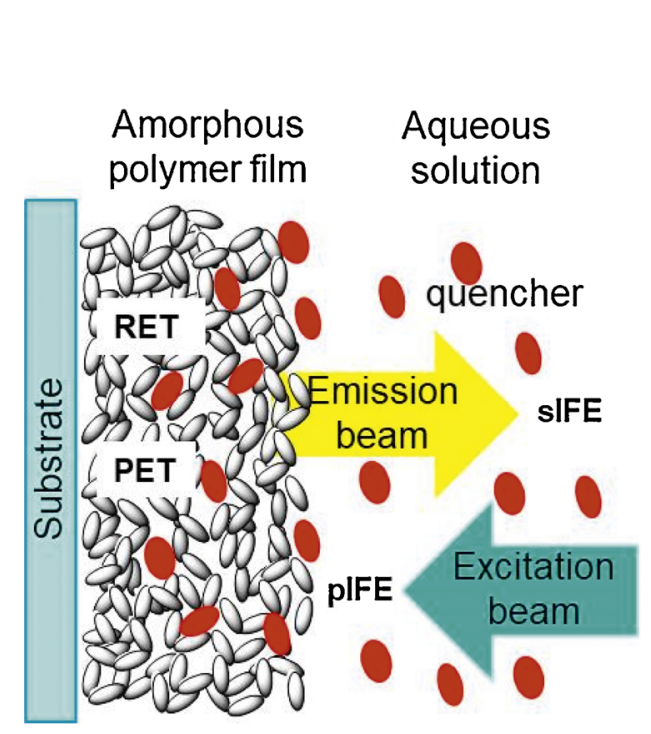

a)

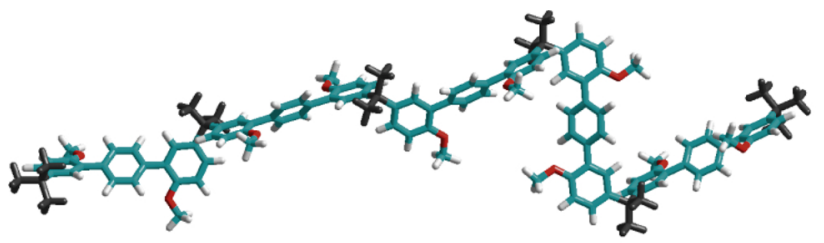

b)

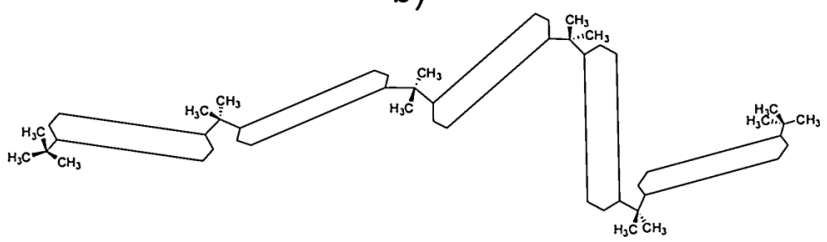

c)

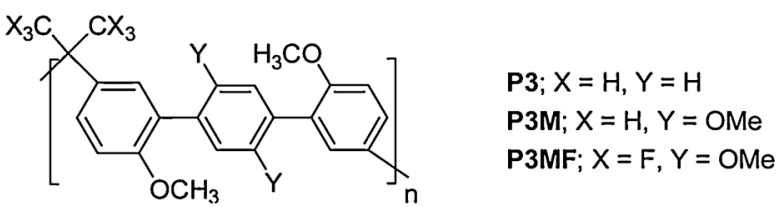

d)

Scheme 1. a) Schematic illustration of the film sensing configuration. b) DFT/PBEh-3c relaxed geometry of a pentamer of P3 showing some of the conformations that the rigid terphenylene chromophores could adopt. c) Sketch of the P3 pentamer. d) Chemical structures of the investigated p-terphenylene polymers.

photoluminescence quenching of fluorescent polymers with electronrich $\pi$-systems by electron-poor nitroaromatic compounds is usually caused by photoelectron transfer (PET) from the LUMO of the photoexcited polymer to the LUMO of the fundamental state of the NAC [4]. Non-radiative depletion of excited states could also occur by radiationless resonance energy transfer (RET) which depends on the extent of spectral overlap between the quencher absorption spectrum and the emission spectrum of the sensing material [1,2]. So, the overall quenching response generally results from either PET [22-24,25] or an association of PET and RET [11,26]. Besides, inner filter effects (IFE) could enhance sensing responses though they cannot rigorously be considered a fluorescence quenching process. This last phenomenon is gaining much attention for the detection for absorbing analytes and in our case occurs when the non-fluorescent nitroaromatic quencher absorbs either the excitation beam (primary IFE, i.e., pIFE) or the fluorophore emission (secondary IFE, i.e., sIFE) as shown in Scheme 1a [27]. Indeed, the selective detection of the strongly absorbing picric acid, PA, has been occasionally attributed entirely to IFE [28] or more frequently to IFE coupled with actual quenching mechanisms [15,29-31].

We have been interested in developing fluorescent film sensors based on new segmented conjugated polymers for NACs detection in water media. Thus, our group has reported on fluorescent segmented conjugated polymers which present high solubility, amorphous morphologies with no aggregation of chromophores in the condensed phase due to the contorted nature of their main chain (see Scheme 1b), excellent film-forming characteristics and good chemosensing abilities [32,33]. In this study, we synthesized a series of new photoactive amorphous segmented conjugated polymers bearing terphenylene chromophores; and evaluated how chemosensing abilities are dependent on structural modifications and sensing experimental conditions, in order to contribute to establishing structureresponse relationships. (Scheme 1d). P3 has methoxy-substituted terphenylene chromophores in the main chain and isopropylene spacers as saturated linking groups along the main chain. P3M and P3MF have terphenylene groups with additional methoxy substituents in the central aromatic ring that could increase their electron donor properties. In P3MF the substituted terphenylene units are linked by perfluoroisopropylene groups which are known to decrease crystallinity in condensed phases. The new polymers were characterized by gel permeation chromatography (GPC), differential scanning calorimetry (DSC), NMR spectroscopy, UV-Vis spectroscopy, fluorescence spectroscopy and cyclic voltammetry (CV). Finally, we evaluated the chemosensing abilities of the polymeric thin films to detect nitroaromatics in aqueous media.

\section{Experimental part}

\subsection{Methods and instrumentation}

Melting points reported were not corrected. ${ }^{1} \mathrm{H}$ NMR (300 MHz) and ${ }^{13} \mathrm{C}$ NMR $(75 \mathrm{MHz})$ spectra were registered with a Bruker AVANCE III spectrometer at $25^{\circ} \mathrm{C}$. Elemental analyses $(\mathrm{C}, \mathrm{H})$ were made on an EXETER CE-440 instrument. GPC analyses (THF) were carried out using a Waters 600 ; calibration was done using polystyrene standards. Thermal analysis was done on a TA Q20 under nitrogen flow. An optical polarizing microscope (Leitz, Model Ortolux) equipped with a hot stage (Mettler) was also employed to analyze the thermal behavior. UV-vis spectra were acquired with a Cary 60 spectrometer. The absorption spectra were collected either on dilute samples (less than $0.01 \mathrm{~g} / \mathrm{ml}$ ) or thin films cast on quartz plates and placed at a $30^{\circ}$ angle to the incident beam. Steady-state fluorescence spectra were obtained from dilute samples (less than $0.02 \mathrm{mg} / \mathrm{ml}$ ) using an SML AMINCO 4800 spectrofluorimeter keeping the optical densities below 0.1 to minimize aggregation and reduce artifacts introduced by self-absorption in fluorescence. Fluorescence depolarization in thin films was measured with the spectrofluorimeter operating in an L-format. Steady-state fluorescence anisotropy was calculated according to the equation z.lsquo;rz.rsquo; $=I_{v v}-\mathrm{GI}_{v h} /\left(I_{v v}+\mathrm{G} 2 I_{v h}\right)$, where $I_{\text {exc,em }}$ is the emission intensity, $v$ and $h$ are the alignment, vertical or horizontal, of the excitation and emission polarizers, and the term $\mathrm{G}=I_{h v} / I_{h h}$ adjusts for any depolarization originated in the optical system. Reported values are an average of the anisotropy measurements in a range of $c a .60 \mathrm{~nm}$. Cyclic voltammetry $(\mathrm{CV})$ in solution was performed on a Bas Epsilon instrument with a three-electrode setup in a solution of $\mathrm{Et}_{4} \mathrm{NClO}_{4}(0.1 \mathrm{M})$ in $\mathrm{CH}_{2} \mathrm{Cl}_{2}$ at a sweep rate of $100 \mathrm{mV} / \mathrm{s}$. Platinum wire was used as working electrode, a $\mathrm{Pt}$ wire as counter electrode and $\mathrm{Ag}$ wire on which $\mathrm{AgCl}$ had been deposited electrolytically as the reference electrode.

\subsubsection{Fluorescence Quenching Studies}

Thin film spectra were recorded by front-face $\left(30^{\circ}\right)$ detection. Films were cast onto carefully leveled quartz or glass substrates $(2.54 \times 1.1 \mathrm{~cm})$ 
by spreading $0.1 \mathrm{ml}$ of a chloroform solution over the whole area. The solution was allowed to evaporate slowly in a nitrogen-filled box, and the film was kept under reduced pressure for 12 hours at $r$. t.. The thickness of the cast films was measured in at least nine different regions using a UV-visible interferometer (Model F20-UV; Filmetrics, Inc.) with a small spot fiber optic, spot size $=0.2 \mathrm{~mm}$. Quenching experiments were carried out in films inserted down to two-thirds of the height of the cell; which was then filled with water $(2.4 \mathrm{~mL})$. Spectra were acquired by front-face detection at $30^{\circ}$ after the addition of microliter aliquots of various NACs. The occurrence of polymer leakage from the film towards the aqueous media was checked during 24 hours by fluorescence control experiments. P3, P3M, and P3MF films supported on quartz or glass plates showed no bleeding.

\subsubsection{Molecular Modeling}

Calculations were made with the ORCA 4.0 program package [34]. Pre- and post-processing operations were done with the graphical interface Gabedit 2.4.8 [35]. Geometries were optimized first with the AM1 method and then refined with the PBEh-3c method [36]. This DFT method is based on a modified PBE functional, uses Ahlrichs-DZ basis sets and the " $3 \mathrm{c}$ " stands for the use of the dispersion correction D3, a gCP correction, and modifications of the double zeta basis sets. Single point energies were calculated using a DFT/PBEh-3c//DFT/PBE0-D3gCP/def2-TZVP protocol. The Conductor-like Polarizable Continuum Model (C-PCM) was used to correct for solvation effects throughout all calculations [37].

\subsection{Materials and synthesis}

Bisphenol A, Bisphenol AF, and 1,4-Benzene diboronic acid were purchased from Aldrich and used as received. THF was purified by distillation from $\mathrm{Na}$ /benzophenone. $\mathrm{Pd}_{2}(\mathrm{dba})_{3}$ was prepared according to the literature [38]. TNT was recrystallized from ethanol.

\subsubsection{Monomer synthesis}

The synthesis of 2,2-bis-(2-bromo-4-methoxyphenyl)propane, 1, and 2,5-dimethoxyphenyl-1,4-bis(trimethyleneboronate), 6, have been already reported [39].

2.2.1.1. 2,2-Bis-(2-bromo-4-methoxyphenyl)perfluoropropane (2). This compound was obtained in two steps. First, a well-stirred mixture of 2bis-(4-hidroxyphenyl)perfluoropropane $\left(3.36 \mathrm{~g}, 10.0 \mathrm{mmol}\right.$ ) and $\mathrm{K}_{2} \mathrm{CO}_{3}$ $(3.50 \mathrm{~g}, 25.0 \mathrm{mmol})$ in acetone $(12 \mathrm{~mL})$ was heated under reflux for $1 \mathrm{~h}$. Then, after cooling to $30-35^{\circ} \mathrm{C}$, methyl iodide $(4.00 \mathrm{~g}, 28.0 \mathrm{mmol})$ was added, and the suspension was maintained at $30-35^{\circ} \mathrm{C}$ for 3 days. Water $(20 \mathrm{~mL})$ was then added, the mixture stirred for $10 \mathrm{~min}$ at room temperature and extracted with $\mathrm{CHCl}_{3}$ (three times $10 \mathrm{~mL}$ ). The combined organic layers were washed with water, aqueous $\mathrm{NaOH}$, aqueous $\mathrm{HCl}$ and water, dried with $\mathrm{Na}_{2} \mathrm{SO}_{4}$ and the solvent was removed under reduced pressure. 2,2-bis(4-methoxyphenyl)perfluoropropane was obtained as a dense liquid after purification by column chromatography on silica gel using hexane/ $\mathrm{CH}_{2} \mathrm{Cl}_{2}$ as eluent. Yield: $3.04 \mathrm{~g}(83 \%) .{ }^{1} \mathrm{H}$ NMR $\left(\mathrm{CDCl}_{3}\right): \delta=7.31\left(\mathrm{~d}, 2 \mathrm{H}, J_{o}\right.$ $=9.16 \mathrm{~Hz}), 6.87\left(\mathrm{~d}, 2 \mathrm{H}, J_{o}=9.16 \mathrm{~Hz}\right), 3.81(\mathrm{~s}, 3 \mathrm{H}) .{ }^{13} \mathrm{C} \mathrm{NMR}\left(\mathrm{CDCl}_{3}\right)$ : $\delta=160.2,131.8,124.8\left(\mathrm{q},{ }^{1} J_{C-F}=286.7 \mathrm{~Hz}\right), 113.9,56.1$.

Then, $\mathrm{Br}_{2}(2.74 \mathrm{~g}, 17.2 \mathrm{mmol})$ was added drop-wise over a period of $40 \mathrm{~min}$ to a solution of 2,2-bis-(4-methoxyphenyl)propane $(2,84 \mathrm{~g}$, $7.8 \mathrm{mmol})$ in $\mathrm{CH}_{2} \mathrm{Cl}_{2}$ at $0{ }^{\circ} \mathrm{C}(6 \mathrm{~mL})$. The mixture was stirred for $5 \mathrm{~h}$ followed by the addition of a $10 \%$ aqueous $\mathrm{NaOH}$ solution $(15 \mathrm{~mL})$. The mixture was then extracted with $\mathrm{CH}_{2} \mathrm{Cl}_{2}$ (three times $5 \mathrm{~mL}$ ), the organic layer was dried with $\mathrm{Na}_{2} \mathrm{SO}_{4}$ and the solvent was removed under reduced pressure. Compound $\mathbf{2}$ was obtained as a very dense liquid after purification by column chromatography on silica gel using hexane $/ \mathrm{CH}_{2} \mathrm{Cl}_{2}$ as eluent. Yield: $3.66 \mathrm{~g}(90 \%) .{ }^{1} \mathrm{H} \mathrm{NMR}\left(\mathrm{CDCl}_{3}\right): \delta=7.54(\mathrm{~s}, 2 \mathrm{H}), 7.30$ (d, $\left.2 \mathrm{H},{ }^{1} J_{C-F} 8.77\right), 6.88$ (d, $2 \mathrm{H}, J$ 8.77), $3.93(\mathrm{~s}, 6 \mathrm{H}) .{ }^{13} \mathrm{C} \mathrm{NMR}\left(\mathrm{CDCl}_{3}\right)$ : $\delta=156.8$, 135.2, 130.8, 126.6, 111.9, 56.7. $\left(\mathrm{C}_{17} \mathrm{H}_{12} \mathrm{Br}_{2} \mathrm{~F}_{6} \mathrm{O}_{2}\right)$ (522.1): Calcd. C, 39.11; H, 2.32; Found: C, 38.93; H, 2.24.
2.2.1.2. 1,4-bis(4,4,5,5-tetramethyl-1,3,2-dioxaborolan-2-yl)benzene (5) was synthesized according to the literature with slight modifications [40]. A mixture of 1,4-phenylenediboronic acid ( $8.3 \mathrm{mmol}, 1.37 \mathrm{~g}$ ), pinacol $(20.65 \mathrm{mmol}, 2.44 \mathrm{~g})$ methanol $(20 \mathrm{~mL})$ and $\mathrm{SO}_{4} \mathrm{Mg}(22.0 \mathrm{mmol}$, $2.65 \mathrm{~g}$ ), was heated at $30^{\circ} \mathrm{C}$ for $24 \mathrm{~h}$. After this period, methanol was evaporated. The resulting solid was dissolved in $\mathrm{CHCl}_{3}(50 \mathrm{~mL})$, dried with $\mathrm{SO}_{4} \mathrm{Mg}$ and the solvent was removed under reduced pressure. The product was washed with toluene/hexane (2:1) to give white crystals. Yield: $1.71 \mathrm{~g}$ (62\%), mp $239^{\circ} \mathrm{C}$. NMR characterizations were consistent with those previously reported in ref. $40 .{ }^{1} \mathrm{H} \mathrm{RMN}\left(\mathrm{CDCl}_{3}\right): \delta=7.79$ (s, $\left.1 \mathrm{H}\right), 1.34$ (s, $6 \mathrm{H}) .{ }^{13} \mathrm{C} \mathrm{RMN}\left(\mathrm{CDCl}_{3}\right): \delta=134.3,84.2,25.3$.

\subsubsection{Polymer synthesis}

2.2.2.1. Poly(2,2"-dimethoxy-p-terphenyl-5,5"-enoisopropylene (P3). The general procedure used for the synthesis of polymers P3, P3M and P3MF is described in detail for P3. A $50 \mathrm{~mL}$ Schlenk tube was charged with 2,2-bis(4-methoxy-3-bromophenyl)propane (0.450 g, $1.08 \mathrm{mmol}$ ), 1,4-bis(4,4,5,5-tetramethyl-1,3,2-dioxaborolan-2-yl)benzene $\quad(0.357 \mathrm{~g}$ $1.08 \mathrm{mmol},), \mathrm{Pd}_{2}(\mathrm{dba})_{3}(0.040 \mathrm{~g}, 0.044 \mathrm{mmol}),, \mathrm{P}(o \text {-tolil })_{3}(0.081 \mathrm{~g}$, $0.26 \mathrm{mmol}$ ), $\mathrm{Na}_{2} \mathrm{CO}_{3}(1.41 \mathrm{~g}, 13.26 \mathrm{mmol}$ ) $)$ and a magnetic stirring bar. The tube was sealed with a rubber septum followed by the filling with Ar atmosphere. Dry THF $(11.0 \mathrm{~mL})$ and water $(11.0 \mathrm{~mL})$ was added with a syringe. The mixture was heated at $80^{\circ} \mathrm{C}$ for 7 days. The reaction mixture was poured into methanol $(30 \mathrm{~mL})$. The resulting precipitate was collected by filtration, and dissolved in $\mathrm{CHCl}_{3}(4 \mathrm{~mL})$. The solution was filtered, evaporated and poured into methanol $(30 \mathrm{~mL})$. The solid was then reprecipitated twice from $\mathrm{CHCl}_{3} /$ methanol. The precipitate was collected by filtration, washed with acetone, and dried in vacuo. Yield $=0.27 \mathrm{~g}(76 \%)$. GPC (THF, PS standards): $M_{\mathrm{n}}=2700 \mathrm{Da}, M_{\mathrm{w}}$ $=4100 \mathrm{Da} .{ }^{1} \mathrm{H} \mathrm{RMN}\left(\mathrm{CDCl}_{3}\right): \delta 7.52(\mathrm{~s}, 2 \mathrm{H}), 7.29\left(\mathrm{~d}, 1 \mathrm{H} J_{m}=2.28 \mathrm{~Hz}\right)$, $7.16\left(\mathrm{~d}, 1 \mathrm{H}, J_{o}=8.39 \mathrm{~Hz}\right), 6.86\left(\mathrm{~d}, 1 \mathrm{H}, J_{o}=8.39 \mathrm{~Hz}\right), 3.78(\mathrm{~s}, 3 \mathrm{H})$, 1.71 (s, $3 \mathrm{H}) .{ }^{13} \mathrm{C}$ RMN $\left(\mathrm{CDCl}_{3}\right): \delta 154.8,143.5,137.7,130.2,129.9$, 129.5, 127.1, 111.1, 55.9, 42.2, 31. $\left(\mathrm{C}_{23} \mathrm{H}_{22} \mathrm{O}_{2}\right)_{n}(330.4)_{n}$ : Calcd. C, 83.60; H, 6.71; Found: C, 83.29; H, 6.54

\subsubsection{Poly(2,2',5',2"-tetramethoxy-p-terphenyl-5,5"-ylene)propylene} (P3M). Yield $=0.25 \mathrm{~g}(59 \%)$. GPC (THF, PS standards): $M_{\mathrm{n}}=7080 \mathrm{Da}$, $M_{\mathrm{w}}=9950 \mathrm{Da}$. The ${ }^{13} \mathrm{C}$ and ${ }^{1} \mathrm{H}$ NMR spectra have been reported [39].

\subsubsection{Poly (2,2',5',2"-tetramethoxy-p-terphenyl-5,5"-ylene)}

perfluoroisopropylene (P3MF). Yield $=0.25 \mathrm{~g}$ (47\%). GPC (THF, PS standards): $M_{\mathrm{n}}=5800 \mathrm{Da}, M_{\mathrm{w}}=7950 \mathrm{Da} .{ }^{1} \mathrm{H} \mathrm{NMR}\left(\mathrm{CDCl}_{3}\right): \delta=7.42$ (d, $J=8.59 \mathrm{~Hz}, 1 \mathrm{H}), 7.20(\mathrm{~s}, 1 \mathrm{H}), 6.94(\mathrm{~d}, J=8.59 \mathrm{~Hz}, 1 \mathrm{H}), 6.84(\mathrm{~s}$, $1 \mathrm{H}), 3.79$ (s, $3 \mathrm{H}), 3.59$ (s, $3 \mathrm{H}) .{ }^{13} \mathrm{C} \mathrm{NMR}\left(\mathrm{CDCl}_{3}\right): \delta=157.1150 .8$, 134.0, 130.54, 127.0, 125.0, 124.6 (c, $\left.{ }^{1} J_{C-F}=286.2 \mathrm{~Hz}\right), 115.8,110.4$, $63.8\left(\mathrm{~m},{ }^{2} J_{C-F}=25.24 \mathrm{~Hz}\right), 56.5$, 55.7. $\left(\mathrm{C}_{25} \mathrm{H}_{20} \mathrm{~F}_{6} \mathrm{O}_{4}\right)_{n}(498.4)_{n}$ : Calcd. C, 60.24; H, 4.04; Found: C, 59.81; H, 3.77.

\section{Results and discussion}

\subsection{Synthesis and thermal properties}

The synthesis of the dibromides and diboronic esters, as well as the polymer syntheses, are outlined in Scheme 2. First, dimethylation and subsequent dibromination of bisphenol derivatives afforded dibromides $\mathbf{1}$ and $\mathbf{2}$ in 70\% and 75\% over the two steps. Pinacol esterification of $\mathbf{3}$ in methanolic media gave 5 in good yield (60\%) while the diboronic ester 6 was obtained by a reported procedure [39]. The polymers were synthesized by Suzuki cross-coupling polycondensation in $\mathrm{THF}-\mathrm{H}_{2} \mathrm{O}$ using a mild base and $\mathrm{Pd}_{2}(\mathrm{dba})_{3} /$ tris(o-tolyl)phosphine as the catalystligand system. The terphenylene-based polymers were obtained in good yields (50-70\%) after two reprecipitations from methanol/ $\mathrm{CHCl}_{3}$. GPC measurements (PS standards, THF) show that they presented reasonable molecular weights, $M_{w}$, good degree of polymerizations, $X_{w}$, and narrow molecular-weight dispersities, $D_{\mathrm{M}}$, that is, P3 $\left(M_{\mathrm{w}}=4100 \mathrm{Da}\right.$, $\left.X_{\mathrm{w}}=12, \oplus_{\mathrm{M}}=1.52\right)$, P3M $\left(M_{w}=9950 \mathrm{Da}, X_{w}=25, \oplus_{\mathrm{M}}=1.41\right)$ and 


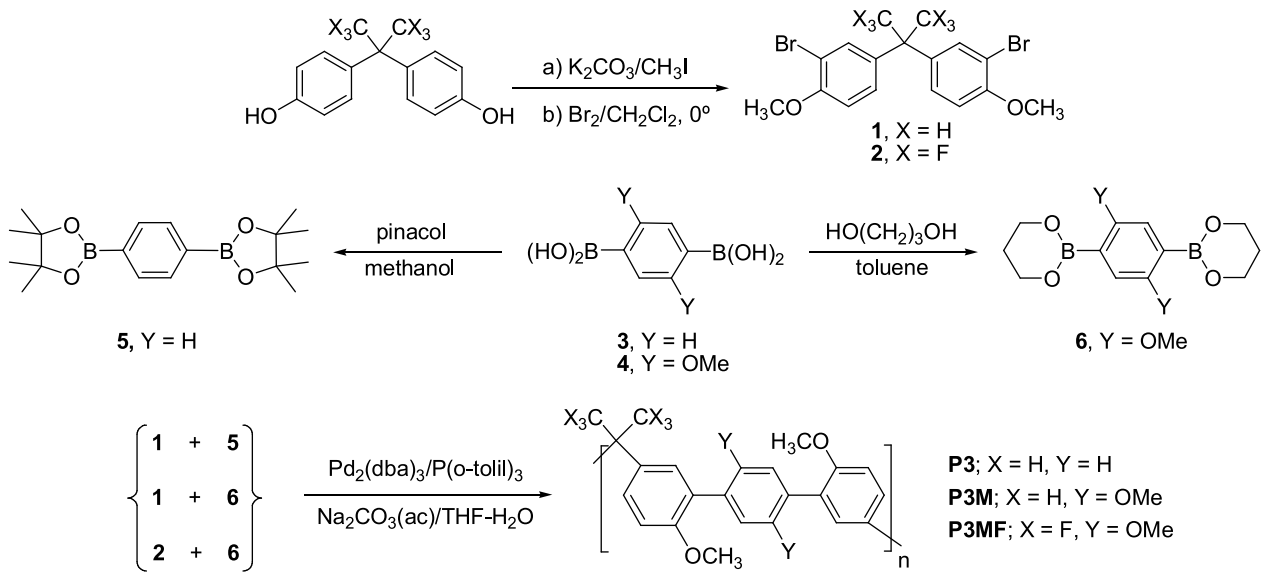

Scheme 2. Synthetic routes of monomers and polymers.

P3MF $\left(M_{w}=7950 \mathrm{Da}, X_{w}=16, \bigoplus_{\mathrm{M}}=1.37\right) .{ }^{1} \mathrm{H}$ and ${ }^{13} \mathrm{C}$ NMR spectroscopy confirmed the structural regularity of the purified polymers (see Supporting information, Fig. S1). All three segmented conjugated polymers were highly soluble in $\mathrm{CHCl}_{3}$ and soluble in $\mathrm{CH}_{2} \mathrm{Cl}_{2}$ and THF. Films deposited on glass or quartz plates by drop-cast using chloroform solutions gave transparent films suitable for optical measurements. No birefringence was observed by POM using cross polarizers in the 50$300{ }^{\circ} \mathrm{C}$ temperature range showing the occurrence of only optically isotropic phases; besides, DSC analyses confirmed that the polymers were completely amorphous. Thus, their DSC traces acquired up to $300{ }^{\circ} \mathrm{C}$ showed only glass transition temperatures $\left(\mathrm{T}_{\mathrm{g}}\right)$ in cooling and heating cycles (Table 1 ). The high values of the $\mathrm{T}_{\mathrm{g}}$ 's of the polymers in the 160 to $180^{\circ} \mathrm{C}$ range can be attributed to the absence of flexible chains in their microstructure as well as to the high content of rigid terphenylene units compared to that of the conformationally rich saturated isopropylene spacers. In an early study, we reported on the amorphous nature of the P3M polymer.[39] Thus, regardless the structural differences, the conformational disorder introduced by the isopropylene spacer renders completely disordered morphologies of P3 and P3MF as well.

\subsection{Electrooptical properties}

The optical properties of the segmented conjugated polymers were measured in both chloroform solutions and as thin films and the corresponding photophysical data is summarized in Table 1 . The absorption spectra of the three polymers in dilute chloroform solution and in the solid state have analogous features. As seen in Fig. 1, the absorption maxima of P3, P3M, and P3MF appeared in a narrow range $(\approx 300$ $310 \mathrm{~nm}$ ) despite their structural differences. Moreover, both solution and solid-state spectra of each polymer nearly superimpose indicating that electronic interaction between neighboring chromophores in the solid state is minimal and resembles, therefore, the isolation of chromophores proper of solution environments. We conclude that the twisted polymer main chain efficiently prevents the chromophore units from forming ground-state aggregates. Fig. 1 also shows the fluorescence spectra of chloroform solutions and the thin films of the three polymers. Their emission maxima appeared in a narrow range $(\approx 370$ $380 \mathrm{~nm}$ ) but show different patterns in their vibronic structure. All polymers in solution presented fluorescent spectrum with almost no vibronic structure. However, in the solid state they differ in the relative intensities of vibronic bands. Thus, the P3MF emission spectrum shows the $0-0$ transition as a faint vibronic shoulder in the blue flank of the $0-1$ peak, and such shoulder is more prominent in the case of P3M. Finally, P3 presents two well defined vibronic bands corresponding to the $0-0$ and $0-1$ peaks. The increase in the $0-0$ to $0-1$ peak intensity ratio has been attributed to emission from a disordered system with growing amounts of weakly aggregated species $[41,42,43]$. Therefore, although the three polymers are disordered at the macroscopic level as determined by DSC, they present various degrees of local order as it can be inferred from photoexcitation experiments. Thus, the increasing amount of locally ordered chromophoric aggregates present in the films follows the sequence P3 $>$ P3M $>$ P3MF. Table 1 also lists the residual value of the steady-state anisotropy measured in thin films by

Table 1

Thermal and optoelectronic properties of the terphenylene polymers.

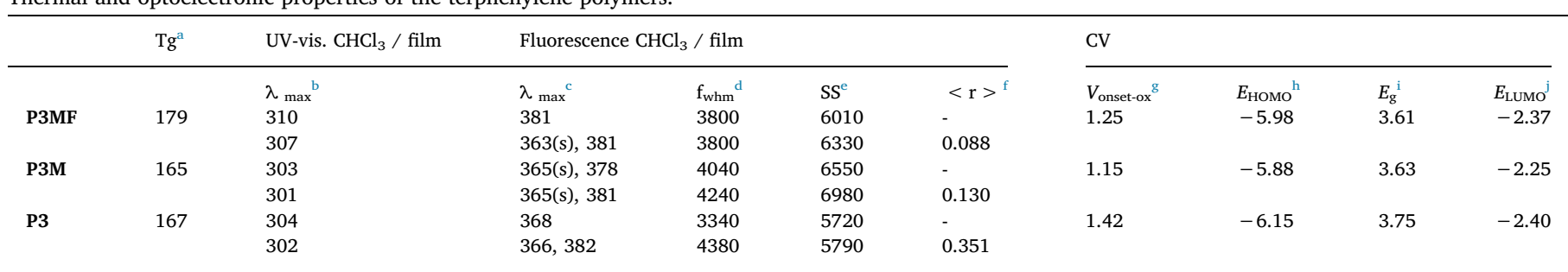

a Glass transition temperature $\left({ }^{\circ} \mathrm{C}\right)$ determined by DSC at scan rates of $5^{\circ} \mathrm{C} / \mathrm{min}$.

b Absorption maxima measured in dilute $\mathrm{CHCl}_{3}$ solutions and on films.

c Emission maxima measured in dilute $\mathrm{CHCl}_{3}$ solutions and on films $\left(\lambda_{\text {exc }}=310 \mathrm{~nm}\right)$.

d Full width at half-maximum of the fluorescence bands (in $\mathrm{cm}^{-1}$ ).

e Stokes shifts $\left(\lambda_{\text {max,em }}-\lambda_{\text {max,abs; }}\right.$ in $\left.\mathrm{cm}^{-1}\right)$.

${ }^{\mathrm{f}}$ Average anisotropy measured in thin films $\left(\lambda_{\mathrm{exc}}=330 \mathrm{~nm}\right)$.

$\mathrm{g}$ Polymer oxidation onset, in $\mathrm{eV}$.

${ }^{\text {h }} E_{\text {HOMO, in eV. }}$

i The optical HOMO-LUMO energy gap based in the low energy onset in the UV-vis spectra, in eV.

j $E_{\text {Lumo, in }} \mathrm{eV}$. 

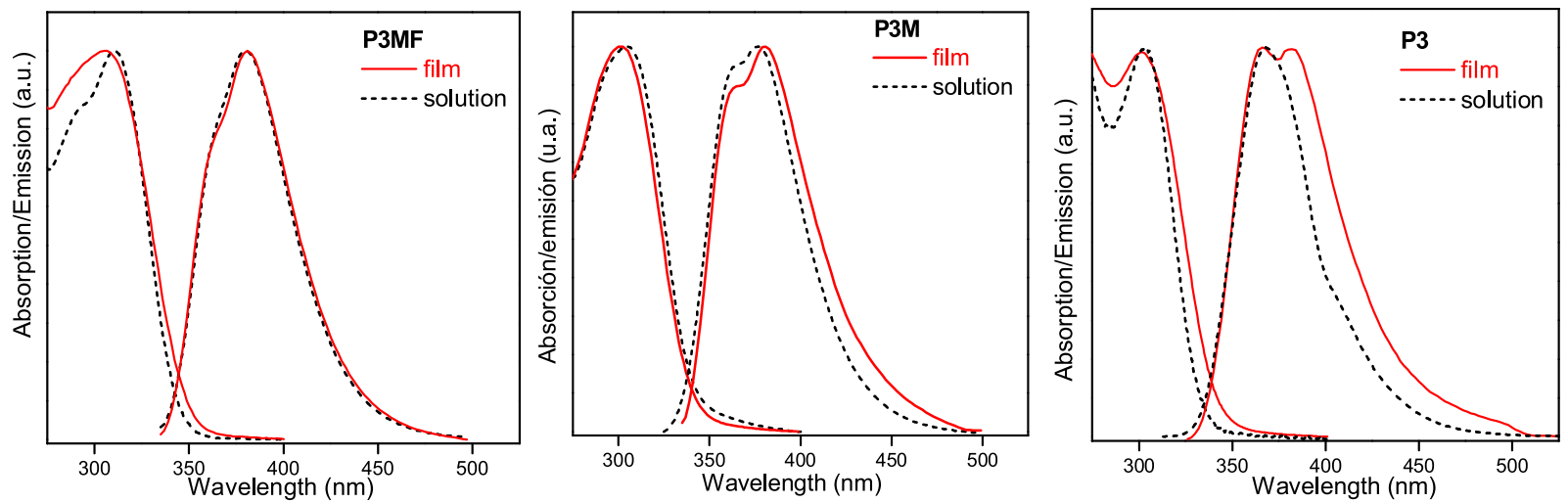

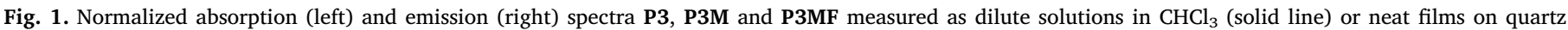
(dashed line).

fluorescence depolarization experiments which are an indication of the exciton mobility through homo resonance energy transfer [21].

Cyclic voltammetry determinations in solution showed that P3M and P3MF experience chemically reversible oxidations while P3 showed an irreversible oxidative process (Fig. S2). The reducing power of the polymer excited states was estimated from their LUMO energies that were calculated from the onset of the oxidation potentials, $E_{\text {ox,onset, }}$ obtained in solution. First, the assumption that the energy level of $\mathrm{Ag}$ / $\mathrm{Ag}+$ is at $4.73 \mathrm{eV}$ below the vacuum level [44] permitted to calculate the HOMO energies. Then, the LUMO energies were obtained from the values of the optical band gaps and the HOMO level energies (Table 1). Thus, we found that the LUMOs were in the range -2.2 to $-2.4 \mathrm{eV}$, showing that all these polymers present good reducing power towards nitroaromatics by photoelectron transfer from the high-energy LUMO of the polymer to the low-energy LUMO of TNT $(-3.7 \mathrm{eV})$ and PA $(-3.89 \mathrm{eV})$ [45]. The DFT results of the calculations performed on trimers follows closely the tendency observed in the experimental determinations (Fig. 2), though discrepancies are observed, as usual, between the absolute values of both sets [46].

\subsection{Photoluminescence quenching}

The quenching studies were performed in aqueous media giving the intended environmental applications. Thus, glass-supported thin films of the polymer were immersed in water, and then the quenching experiments were carried out by varying the concentration of NACs.

Fig. 3a shows the steady decrease of the fluorescence intensity of a thin film of P3MF which was irradiated with an excitation wavelength $\left(\lambda_{\text {exc }}\right)$ of $330 \mathrm{~nm}$. The quenching of the fluorescence was achieved by adding aliquots of an aqueous solution of TNT with concentrations in the nanomolar and micromolar range. The absorption spectra of TNT and their overlaps with the absorption and emission spectra of P3MF are shown in Fig. 3b. It can be appreciated there that the overlap of TNT with the absorption of P3MF is quite modest at $330 \mathrm{~nm}$ but becomes more prominent at smaller wavelengths. Fig. $3 \mathrm{c}$ shows the quenching efficiency plots, $\mathrm{QE}=1$ - I/I0, of P3MF with TNT which were obtained with three increasingly shorter $\lambda_{\text {exc }}$, i.e., $330 \mathrm{~nm}, 300 \mathrm{~nm}$, and $285 \mathrm{~nm}$. As expected, we found that quenching efficiency depends upon the magnitude of the molar extinction coefficient $\varepsilon$ of the quencher TNT at each excitation wavelength. Fig. $3 c$ shows that the response at $\lambda$ exc $=330$ is the smaller while the one at $\lambda$ exc $=285 \mathrm{~nm}$ is the larger. These results indicate that the polymer sensitivity can be enhanced by the judicious selection of the excitation wavelength. This higher efficiency would probably be due to an increase of the IFE contribution to the overall response since a contribution from RET quenching can be disregarded on account of the minimal overlap of the absorption spectrum of TNT and the emission spectrum of P3MF. Similarly, PET quenching cannot be responsible for enhancing the quenching efficiency because an excitation

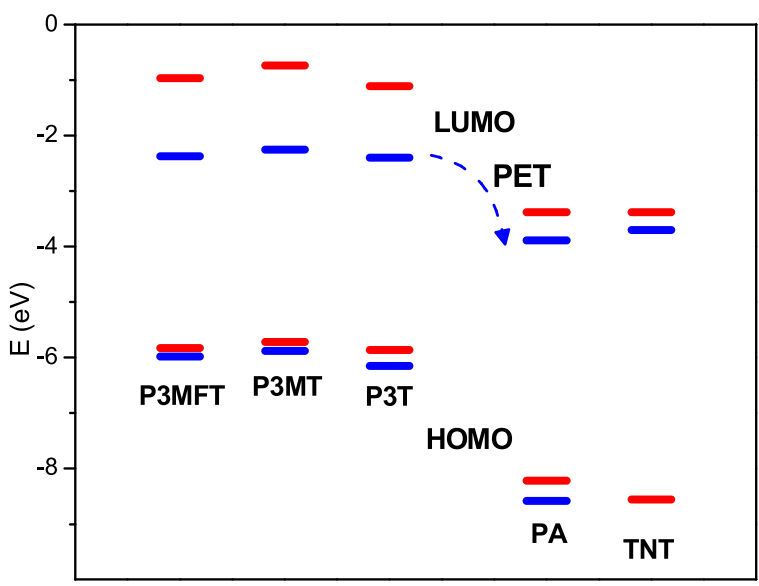

Fig. 2. Frontier molecular orbitals energies of trimers (P3MFT, P3MT and P3T). Experimental (blue). Calculated by DFT (red). Values for the FMOs of TNT and PA are also included.

wavelength dependence of the type and amount of the emitting species responsible for PET quenching cannot be expected in photoluminescence excitation experiments (Kasha-Vavilov's rule).

Interestingly, the quenching regime for TNT presents two distinct regions as we observed previously with a structurally different polymer [33]. Thus, the Stern-Volmer plots of P3MF and TNT in Fig. 4 display a rapid increase of the quenching efficiency (see also the inset in Fig. 4a) followed by an inflection point around $5 \mu \mathrm{M}$, and then grow at a slower pace in a quasi-linear fashion till the end of the studied region $(50 \mathrm{nM}$ $400 \mu \mathrm{M}$, Fig. S3). We now tentatively associated the initial steep increase of the quenching efficiency in the nanomolar and low micromolar range to quenching interactions either on the film surface [47] or, more probably, within an ultrathin surface layer of the order of a few nanometers. Experimental [48] and theoretical [49] studies have indicated that polymer-supported thin films had a soft interfacial layer of $\approx 10 \mathrm{~nm}$ thickness with reduced density and greater mobility of polymer chains $[50,51]$. These particular characteristics, in conjunction with the plasticizing effect of the solvation, which by diffusion of the solvent molecules into the amorphous material also reduces the glass transition and increase the chain mobility, could create a soft sensing region in direct contact with the analyte solution where the diffusional quencher intake by this softer layer is facilitated thus resulting in an increase of the interaction between quencher and chromophoric units.

Furthermore, to evaluate the relative influence of the IFE in the P3MF response the fluorescence intensities were registered at 1, 10, 20 and $30 \mathrm{~min}$ after the addition of each aliquot of the quencher solution. The S-V plot showed in Fig. 4a indicates that the response of a P3MF film towards TNT when it is irradiated at $\lambda_{\text {exc }}=285 \mathrm{~nm}$ increases 

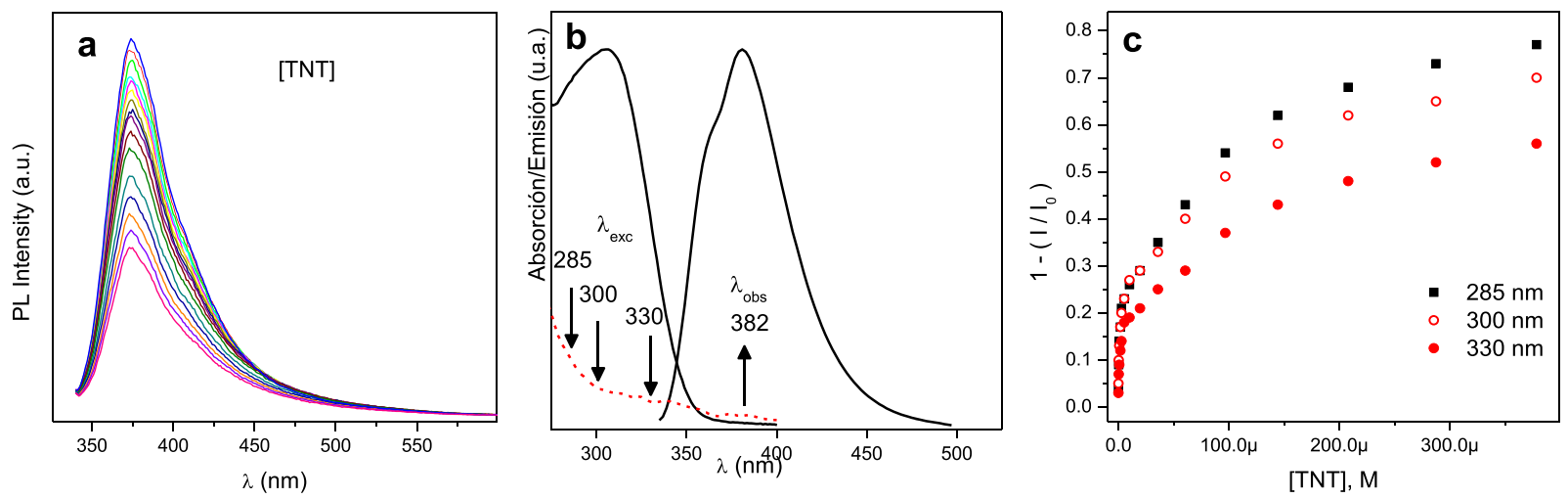

Fig. 3. (a) Fluorescence spectra change $\left(\lambda_{\mathrm{ex}}=330 \mathrm{~nm}\right.$ ) of P3MF films (thickness $\approx 35 \mathrm{~nm}$ ) as a function of added TNT in water; [TNT] $=0.05-279 \mu \mathrm{M}$ (top to bottom). b) Absorption spectrum of the TNT in water (red dashed line). Absorption spectrum of a P3MF film (solid line) and fluorescence spectra $\left(\lambda_{\text {exc }}=330 \mathrm{~nm}\right.$ ). Down arrows indicate the $\lambda_{\text {exc }}$ used to gather data presented in (c) while the up arrow indicates the $\lambda_{\text {em }}$ used to observe the fluorescence decrease. c) The quenching efficiencies for a P3MF film $\left(\lambda_{\text {obs }}=382 \mathrm{~nm}\right)$ and TNT obtained with $\lambda_{\text {excitation }}=285,300$ and $330 \mathrm{~nm}$.

gradually after each aliquot addition, implying that the quencher penetrates slowly into the film to produce a diffusion-controlled quenching phenomenon. The decay of the fluorescence intensity slowed down with time so the quenching efficiency values at $20 \mathrm{~min}$ and 30 min were rather close. Notably, there were appreciable jumps in quenching efficiency immediately after the addition of the next TNT aliquot, which are more evident at higher concentrations and do not occur in the nanomolar range (see inset in Fig. 2a); so these sudden increases could be attributed to IFE. Thus, for a quenching of $53 \%$ of the initial fluorescence, $I_{0}$, diffusion-controlled quenching contributions sum up to $\approx 37 \%$ (Fig. S4a), leaving then an IFE contribution of $\approx$ $16 \%$. Therefore the overall quenching efficiency results from initial excitation beam attenuation due to TNT absorption (i.e., pIFE) which is followed by gradual photoluminescence quenching by PET as the quencher diffuses into the film. These results are confirmed by the experiment using a $\lambda_{\text {exc }}$ of $330 \mathrm{~nm}$ where the molar extinction coefficient $\varepsilon$ of TNT is smaller then the one at $\lambda=330 \mathrm{~nm}$ (Fig. 3b). In this case, the fluorescence values recorded $30 \mathrm{~min}$ after the quencher addition at a given concentration are rather similar to the values recorded $1 \mathrm{~min}$ after the addition of the next quencher aliquot. Indeed, for a quenching of $48 \%$ of the initial fluorescence, $\mathrm{I}_{\mathrm{o}}$, diffusion-controlled quenching contributions sum up to $\approx 42 \%$ (Fig. S4b), leaving then a small IFE contribution of $\approx 6 \%$ (Fig. S4b).

The S-V relationship was also employed to evaluate the effect of structural modifications on the sensing properties of the polymers. Fig. 5 shows the S-V plots obtained for the TNT quencher with polymeric films excited at two wavelengths. Both PM3 and P3 display the nonlinear pattern with an inflection point already observed with P3MF. As expected from the fact that the absorption and emission maxima of P3, P3M, and P3MF appeared in a narrow range, the comparison of the
Fig. 5a and $\mathrm{b}$ indicated that the polymers showed a similar increase in sensitivity due to IFE. P3MF showed the larger initial response at nanomolar concentrations. Besides, the quenching response order observed for TNT was P3MF $>$ P3M $>$ P3. Thus, the quenching efficiency order found does not follow the electron donor capability order obtained by cyclic voltammetry, i.e., P3M $>$ P3MF $\approx$ P3. Then, the Hansen's solubility parameter distances, $\mathrm{R} a$, [52] of TNT with P3MF ( $\mathrm{R} a=8.2)$, P3M $(\mathrm{R} a=7.5)$ and P3 $(\mathrm{R} a=11.2)$ were calculated $[53,54]$ in an attempt to relate the observed quenching order to the TNT solubility into the polymers that could facilitate the quencher diffusion into the film. Keeping in mind that the smaller the $\mathrm{R} a$, the higher the analyte-polymer compatibility, the calculated order of TNTpolymer cohesive energies (P3 $<$ P3MF $<$ P3M) does not follow the polymer quenching order. On the other hand, the residual values of the steady-state anisotropy, $\langle\mathrm{r}\rangle$, obtained by fluorescence depolarization experiments (Table 1$)$ indicate that P3MF $(<\mathrm{r}>=0.088)$ displays the larger mobility of the excitons into the polymer film, while energy migration from chromophore to chromophore is somewhat reduced in P3M (z.lsquo;rz.rsquo; $=0.130$ ) and movement of excited states virtually disappeared in P3 (z.lsquo;rz.rsquo; $=0.351$ ); considering that z.lsquo;rz.rsquo; thinsp; $=0.4$ corresponds to a scenario devoid of depolarization [27]. Such results have particular significance considering that polymer sensitivity to analytes is enhanced as mobility of excited states towards quenching sites increase. So, the residual values of the steady-state anisotropy of P3MF, P3M, and P3 suggest that the initial quenching response in these polymer series is primarily dependent on the mobility of the excited states within the film.

We also investigate P3MF luminescence response to PA, whose absorbance is significantly higher than that of TNT, at $\lambda_{\text {exc }}=330 \mathrm{~nm}$, $300 \mathrm{~nm}$, and $285 \mathrm{~nm}$ as shown in Fig. 6a. A general analysis indicates
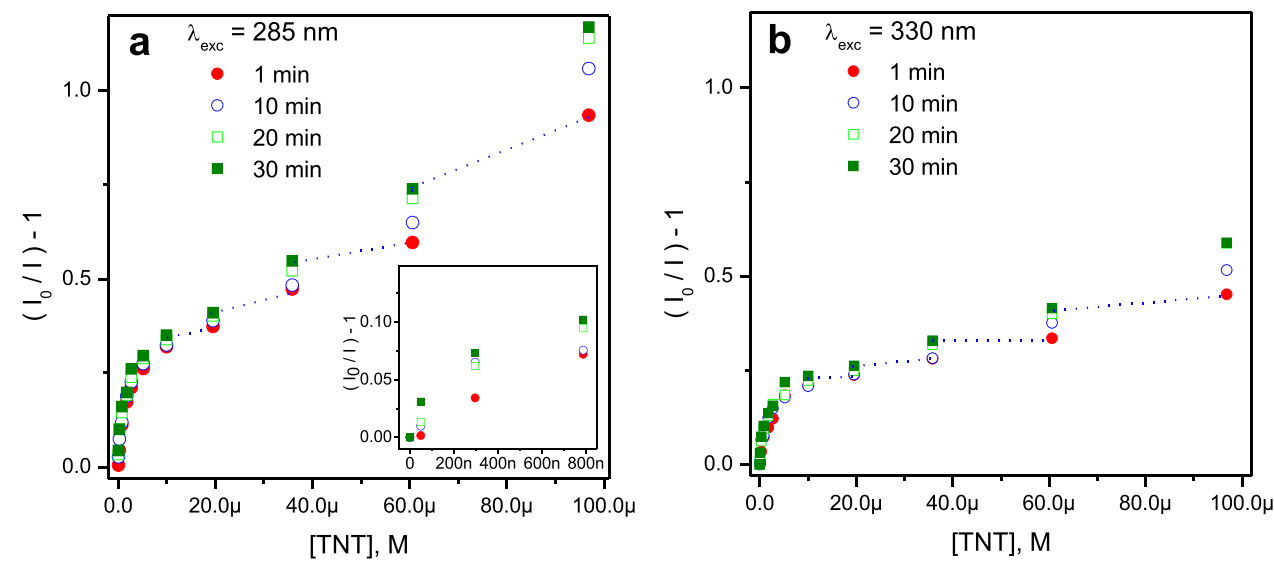

Fig. 4. The Stern-Volmer plots for P3MF films (thickness $\approx 30 \mathrm{~nm}$ ) with TNT irradiated at (a) $\lambda_{\text {exc }}=285 \mathrm{~nm}$, inset show an expansion of the scale close to the origin, and (b) $\lambda_{\text {exc }}$ $=330 \mathrm{~nm}$. Dotted lines are guides to the eye. Dotted lines link the last measurement (after $30 \mathrm{~min}$ ) at a given concentration and the first (after $1 \mathrm{~min}$ ) at the next concentration. 

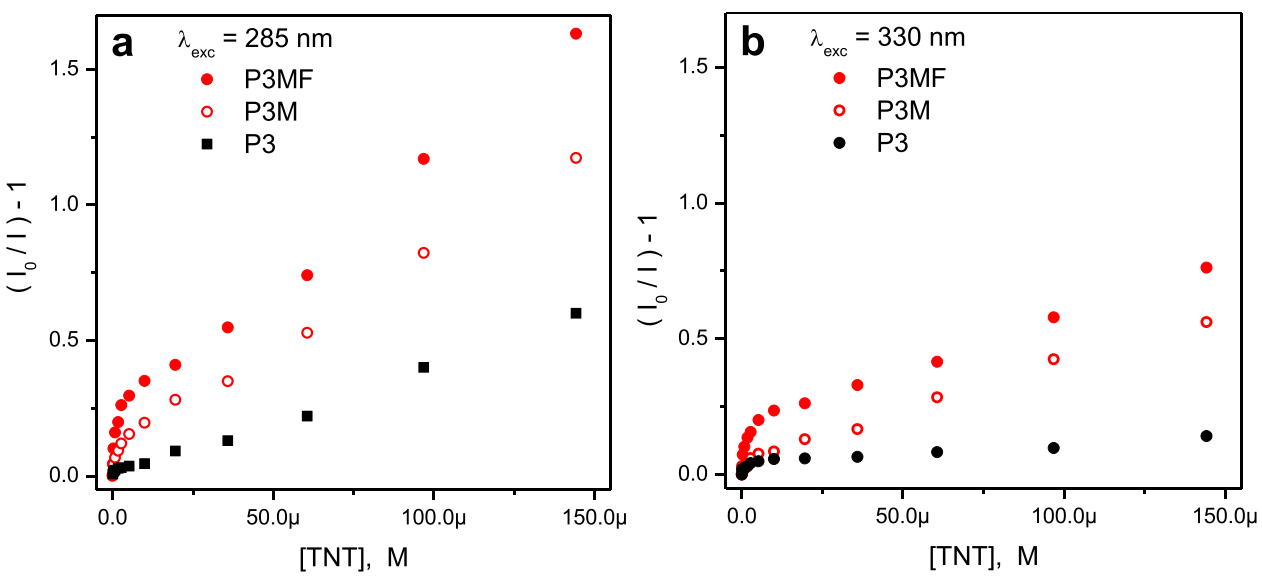

Fig. 5. The Stern-Volmer plots for P3, P3M, and P3MF films (thickness $\approx 33 \mathrm{~nm}$ ) with TNT, (a) $\lambda_{\text {exc }}=285 \mathrm{~nm}$ and (b) $\lambda_{\text {exc }}=330 \mathrm{~nm}$.

firstly that the significant spectral overlap between the absorption spectra of PA and P3MF will produce a primary IFE response dependent upon the absorbance of PA at each excitation wavelength. Secondly, the complete overlap of the absorption spectrum of PA and the emission spectrum of P3MF will generate a strong secondary IFE response whose magnitude will be independent of $\lambda_{\text {exc }}$.

The quenching pattern with PA, Fig. $6 \mathrm{~b}-\mathrm{d}$, conforms to the preceding analysis. First, we observe larger responses with increasing $\lambda_{\text {exc. }}$. Thus, we obtain $48 \%, 53 \%$ and $65 \%$ of the total quenching with a PA solution of $60 \mu \mathrm{M}$ that have absorbancies of $0.174,0.283$ and 0.772 at $330 \mathrm{~nm}$, $300 \mathrm{~nm}$, and $285 \mathrm{~nm}$, respectively. So, primary IFE is probably the factor behind this quasi-linear character of the relationship between response and absorbance (Fig. S5). Indeed, while for TNT the response at $\lambda_{\text {exc }}$
$=285 \mathrm{~nm}$ is larger than the one at $\lambda_{\mathrm{exc}}=330 \mathrm{~nm}$, we observed that the PA response at $\lambda_{\text {exc }}=285 \mathrm{~nm}$ is smaller than that at $\lambda_{\text {exc }}=330 \mathrm{~nm}$, all this as a result of the fact that the larger absorptivities of TNT and PA occur at $285 \mathrm{~nm}$ and $330 \mathrm{~nm}$ respectively. Secondly, in addition to the diffusion-controlled quenching contributions, we see noticeable instantaneous drops in fluorescence intensity in the $\mu \mathrm{M}$ range at all $\lambda_{\text {exc }}$ after PA addition. Thus, for a quenching of $48 \%$ of the initial fluorescence, $\mathrm{I}_{\mathrm{o}}$, we estimated a diffusion-controlled quenching contribution of $\approx 32 \%$ and an IFE contribution of $\approx 16 \%$ when a $\lambda_{\mathrm{exc}}=285 \mathrm{~nm}$ was used (Fig. S4c) while for the experiment using $\lambda_{\text {exc }}=330 \mathrm{~nm}$ (Fig. S4d) the estimated values are $\approx 28 \%$ and $\approx 20 \%$, respectively. These large IFE contributions $\left(\approx 16 \%\right.$ at $\lambda_{\text {exc }}=285 \mathrm{~nm}$ and $\approx 20 \% \lambda_{\text {exc }}=330 \mathrm{~nm}$ ) are due to the combination of a pIFE component and a large sIFE
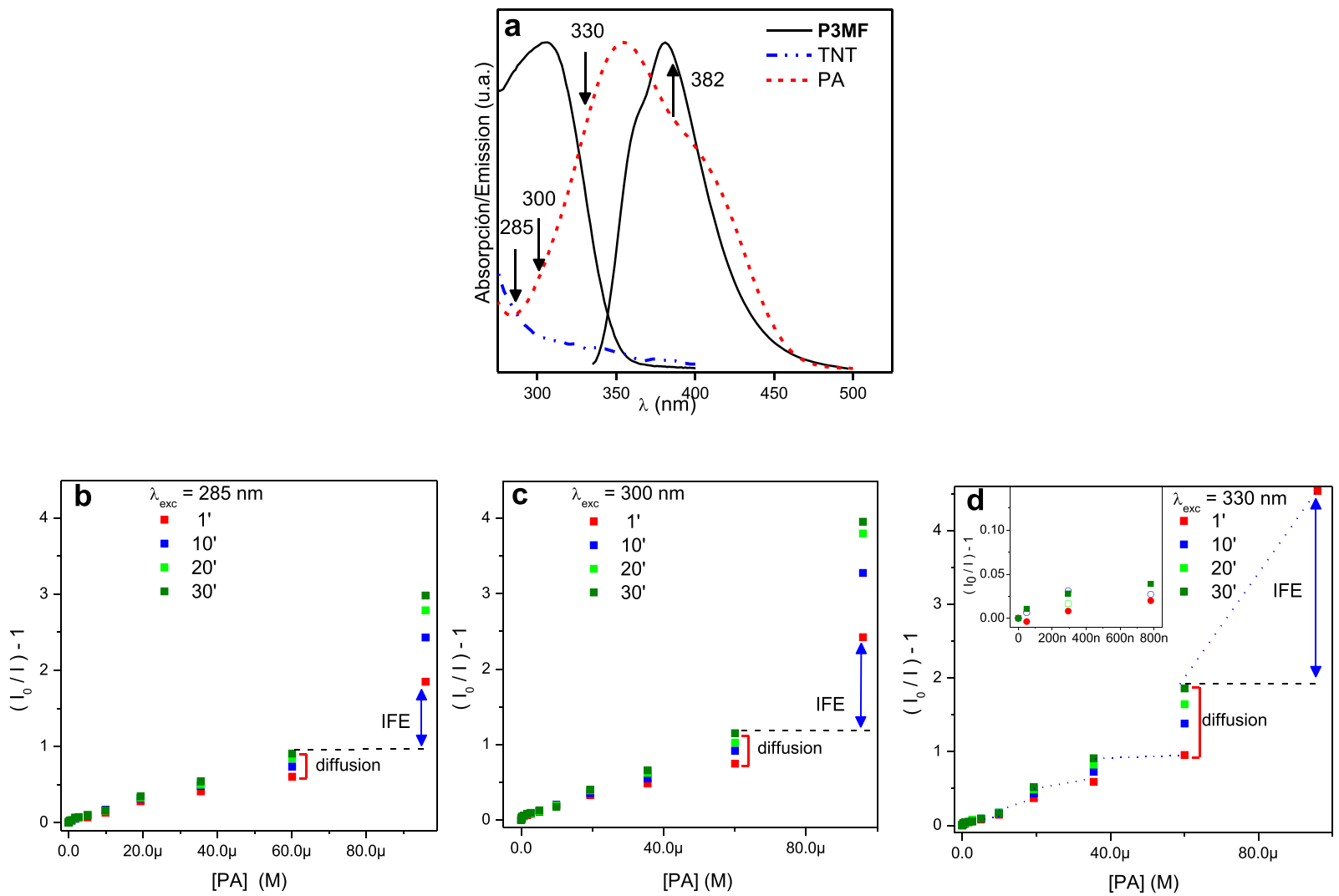

Fig. 6. a) Absorption spectra of PA and TNT in water (equal concentrations). Absorption and fluorescence spectra of a P3MF film. Down arrows indicate the $\lambda_{\text {exc }}$ while the up arrow indicates the $\lambda_{\mathrm{em}}$ used to observe the fluorescence decrease. The Stern-Volmer plots for P3MF films (thickness $\approx 33 \mathrm{~nm}$ ) with PA irradiated at (b) $\lambda_{\text {exc }}=285 \mathrm{~nm}$, (c) $\lambda_{\text {exc }}=300 \mathrm{~nm}$ and (d) $\lambda_{\text {exc }}=330 \mathrm{~nm}$. Dotted lines link the last measurement (after $30 \mathrm{~min}$ ) at a given concentration and the first (after $1 \mathrm{~min}$ ) at the next concentration. Inset show an expansion of the scale close to the origin. 
component, and are rooted in the high absorbance of PA and the overlap of the absorption band of PA and the emission band of P3MF. Therefore the overall quenching efficiency of PA results from excitation and emission beam attenuation due to PA absorption (i.e., pIFE and SIFE) and photoluminescence quenching by PET, and possibly by RET, as the quencher interacts with the polymer film.

In the nanomolar range, only diffusion-controlled quenching is operative because the low absorbance of quencher solutions precludes IFE. Thus, no instantaneous drops in fluorescence intensity after quencher addition can be seen in the insets in Fig. 4a, and Fig. 6d. Notably, the quenching efficiency of PA is lower than that of TNT. We noted that the calculated order of quencher-P3MF compatibility is TNT $(\mathrm{R} a=8.2)>$ PA ( $R a=12.2)$, thus the observed quenching order (TNT $>$ PA) could be related to the ability of the quencher to diffuse into the film.

\section{Conclusions}

Three new photoactive segmented conjugated polymers with terphenylene chromophores were synthesized and the chemosensing abilities to detect nitroaromatics (NACs) of the thin films were evaluated in aqueous media. Their polymeric films are strongly fluorescent, amorphous with no aggregation of the chromophores in the solid state and sensitive towards NACs in water.

Though true quenching, i.e. the depletion of the excited state population of chromophoric units occurring after diffusion of the NACs into the film, contributes to the total response of these polymeric films, the apparent quenching efficiencies of NACs are also influenced by the quencher partial absorption of the excitation beam and/or emission beam. These inner filter effect contributions could be used to increase the film response to the quencher. That is, to maximize the response of these polymer films, it is convenient to use the shortest feasible $\lambda_{\text {exc }}$ for TNT and the longest feasible $\lambda_{\text {exc }}$ for PA. In the micromolar concentration region, the highly absorbing PA frequently has a stronger response than TNT due to the IFE factors. However, we observed that at the nanomolar range where IFE effects are greatly diminished, the properties of the material become more relevant to define quenching efficiencies. Thus, P3MF, which has higher exciton mobility, was more sensitive towards TNT; though the electron-donor capabilities of the chromophores have not bearing on quenching efficiency. Moreover, the TNT > PA quenching order can be attributed to the higher solubility of the TNT quencher into P3MF. So, the tuning of morphology and photophysical properties of the polymer by structural design can be complemented with a rational selection of experimental conditions, e.g., $\lambda_{\text {exc }}$, in order to enhance responses towards the NAC of interest.

\section{Acknowledgments}

Financial support from SGCyT-UNS and CIC-PBA is acknowledged. ABS and MJR thank CONICET for a fellowship. MFA is member of the research staff of CIC-PBA. PGDR and ROG are members of the research staff of CONICET.

\section{Appendix A. Supplementary data}

Supplementary material related to this article can be found, in the online version, at doi:https://doi.org/10.1016/j.jphotochem.2019.112016.

\section{References}

[1] R.P. Schwarzenbach, T. Egli, T.B. Hofstetter, U. Von Gunten, B. Wehrli, Global water pollution and human health, Annu. Rev. Environ. Resour. 35 (2010) 109-136, https://doi.org/10.1146/annurev-environ-100809-125342.

[2] R.P. Schwarzenbach, P.M. Gschwend, D.M. Imboden, Environmental Organic Chemistry, Wiley, New York, 2003, p. 1311 ISBN-10:1118767233.

[3] J. Ye, A. Singh, O.P. Ward, Biodegradation of nitroaromatics and other nitrogencontaining xenobiotics, World J. Microbiol. Biotechnol. 20 (2004) 117-135, https://doi.org/10.1023/B:WIBI.0000021720.03712.12.
[4] X. Sun, Y. Wang, Y. Lei, Fluorescence based explosive detection: from mechanisms to sensory materials, Chem. Soc. Rev. 44 (2015) 8019-8061, https://doi.org/10. 1039/C5CS00496A.

[5] S. Rochat, T.M. Swager, Conjugated amplifying polymers for optical sensing applications, ACS Appl. Mater. Interfaces 5 (2013) 4488-4502, https://doi.org/10. 1021/am400939w.

[6] C.M. Hegguilustoy, R.S. Montani, P.G. Del Rosso, M.J. Romagnoli, R.O. Garay, Highly luminescent anthracene sulfides. Synthesis, experimental and DFT study of their optical properties and interaction with electron deficient nitroaromatic compounds, J. Photochem. Photobiol. A 347C (2017) 67-77, https://doi.org/10.1016/j. jphotochem.2017.07.011.

[7] P. Marks, S. Cohen, M. Levine, Highly efficient quenching of nanoparticles for the detection of electron-deficient nitroaromatics, J. Polymer Sci. Part A: Polym. Chem. 51 (2013) 4150-4155, https://doi.org/10.1002/pola.26824.

[8] J. Huang, J. Gu, Z. Meng, X. Jia, K. Xi, Signal enhancement of sensing nitroaromatics based on highly sensitive polymer dots, Nanoscale 7 (2015) 15413-15420, https://doi.org/10.1039/C5NR01489A.

[9] W. Dong, T. Fei, U. Scherf, Conjugated polymers containing tetraphenylethylene in the backbones and side-chains for highly sensitive TNT detection, RSC Adv. 8 (2018) 5760-5767, https://doi.org/10.1039/C7RA13536J.

[10] T. Han, Y. Zhang, B. He, J. Lam, B. Tang, Functional Poly (dihalopentadiene) s: Stereoselective Synthesis, Aggregation-Enhanced Emission and Sensitive Detection of Explosives, Polymers 10 (2018) 821, https://doi.org/10.3390/polym10080821.

[11] A. Chowdhury, P.S. Mukherjee, Electron-rich triphenylamine-based sensors for picric acid detection, J. Org. Chem 80 (2015) 4064-4075, https://doi.org/10.1021/ acs.joc.5b00348.

[12] I.S. Kovalev, O.S. Taniya, N.V. Slovesnova, G.A. Kim, S. Santra, G.V. Zyryanov, D.S. Kopchuk, A. Majee, V.N. Charushin, O.N. Chupakhin, Fluorescent Detection of 2,4-DNT and 2,4,6-TNT in Aqueous Media by Using Simple Water-Soluble Pyrene Derivatives, Chem. Asian J. 11 (2016) 775-781, https://doi.org/10.1002/asia. 201501310.

[13] S. Joshi, S. Kumari, E. Chamorro, D.D. Pant, R. Sakhuja, Fluorescence Quenching of a Benzimidazolium-based Probe for Selective Detection of Picric Acid in Aqueous Medium, ChemistrySelect 1 (2016) 1756-1762, https://doi.org/10.1002/slct. 201600383.

[14] P. Ghosh, J. Das, A. Basak, S.K. Mukhopadhyay, P. Banerjee, Nanomolar level detection of explosive and pollutant TNP by fluorescent aryl naphthalene sulfones: DFT study, in vitro detection and portable prototype fabrication, Sens. Actuators B 251 (2017) 985-992, https://doi.org/10.1016/j.snb.2017.05.126.

[15] A.S. Tanwar, S. Hussain, A.H. Malik, M.A. Afroz, P.K. Iyer, Inner filter effect based selective detection of nitroexplosive-picric acid in aqueous solution and solid support using conjugated polymer, ACS Sensors 1 (2016) 1070-1077, https://doi.org/ 10.1021/acssensors.6b00441.

[16] X.S. Ma, D.H. Wang, Y.Z. Cui, F.R. Tao, Y.T. Wang, T.D. Li, A novel hydrophilic conjugated polymer containing hydroxyl groups: Syntheses and sensing performance for NACs in aqueous solution, Sens. Actuators B 251 (2017) 851-857, https://doi.org/10.1016/j.snb.2017.05.120.

[17] L. Ding, Y. Fang, Chemically assembled monolayers of fluorophores as chemical sensing materials, Chem. Soc. Rev. 39 (2010) 4258-4273, https://doi.org/10.1039/ C003028G.

[18] W. Guan, W. Zhou, J. Lu, C. Lu, Luminescent films for chemo-and biosensing, Chem. Soc. Rev. 44 (2015) 6981-7009, https://doi.org/10.1039/C5CS00246J.

[19] R. Sun, X. Huo, H. Lu, S. Feng, D. Wang, H. Liu, Recyclable fluorescent paper sensor for visual detection of nitroaromatic explosives, Sens. Actuators B 265 (2018) 476-487, https://doi.org/10.1016/j.snb.2018.03.072.

[20] O. Varnavski, I.D.W. Samuel, L.O. Pålsson, R. Beavington, P.L. Burn, T. Goodson III, Investigations of excitation energy transfer and intramolecular interactions in a nitrogen corded distrylbenzene dendrimer system, J. Chem. Phys. 116 (20) (2002) 8893-8903, https://doi.org/10.1063/1.1471241.

[21] M. Guo, O. Varnavski, A. Narayanan, O. Mongin, J.P. Majoral, M. Blanchard-Desce, T. Goodson III, Investigations of energy migration in an organic dendrimer macromolecule for sensory signal amplification, J. Phys. Chem. A 113 (16) (2009) 4763-4771, https://doi.org/10.1021/jp8112123.

[22] X.S. Ma, Y.Z. Cui, Y.Q. Ding, F.R. Tao, B. Zheng, R.H. Yu, W. Huang, 2D hyperbranched conjugated polymer for detecting TNT with excellent exciton migration, Sens. Actuators B 1238 (2017) 48-57, https://doi.org/10.1016/j.snb.2016.07.025.

[23] X.S. Bandyopadhyay, P. Pallavi, A.G. Anil, A. Patra, Fabrication of porous organic polymers in the form of powder, soluble in organic solvents and nanoparticles: a unique platform for gas adsorption and efficient chemosensing, Polym. Chem. 6 (2015) 3775-3780, https://doi.org/10.1039/C5PY00235D.

[24] G. He, N. Yan, J. Yang, H. Wang, L. Ding, S. Yin, Y. Fang, Pyrene-containing conjugated polymer-based fluorescent films for highly sensitive and selective sensing of TNT in aqueous medium, Macromolecules 44 (2011) 4759-4766, https://doi.org/ 10.1021/ma200953s.

[25] B. Zheng, Y. Li, F. Tao, Y. Cui, T. Li, Enhanced superquenching of the hyperbranched conjugated polymer for the detection of nitroaromatic explosives, Sens. Actuators B 241 (2017) 357-363, https://doi.org/10.1016/j.snb.2016.10.057.

[26] K.R. Ghosh, S.K. Saha, Z.Y. Wang, Ultra-sensitive detection of explosives in solution and film as well as the development of thicker film effectiveness by tetraphenylethene moiety in AIE active fluorescent conjugated polymer, Polym. Chem. 5 (2014) 5638-5643, https://doi.org/10.1039/C4PY00673A.

[27] J.R. Lakowicz, Principles of Fluorescence Spectroscopy, third ed., Springer, New York, 2006.

[28] M. Ganiga, N.P. Mani, J. Cyriac, Synthesis of Organophilic Carbon Dots, Selective Screening of Trinitrophenol and a Comprehensive Understanding of Luminescence Quenching Mechanism, ChemistrySelect 3 (2018) 4663-4668, https://doi.org/10. 
1002/slct.201702646.

[29] C.M. Teixeira, A.I. Costa, J.V. Prata, A new fluorescent double-cavity calix [4] arene: synthesis and complexation studies toward nitroanilines, Tetrahedron Lett. 54 (2013) 6602-6606, https://doi.org/10.1016/j.tetlet.2013.09.109.

[30] B.B. Chen, Z.X. Liu, H.Y. Zou, C.Z. Huang, Highly selective detection of 2, 4, 6trinitrophenol by using newly developed terbium-doped blue carbon dots, Analyst 141 (2016) 2676-2681, https://doi.org/10.1039/C5AN02569A.

[31] A.S. Tanwar, L.R. Adil, M.A. Afroz, P.K. Iyer, Inner Filter Effect and Resonance Energy Transfer Based Attogram Level Detection of Nitroexplosive Picric Acid Using Dual Emitting Cationic Conjugated Polyfluorene, ACS Sensors 3 (2018) 1451-1461, https://doi.org/10.1021/acssensors.8b00093.

[32] P.G. Del Rosso, M.J. Romagnolli, M.F. Almassio, C.A. Barbero, R.O. Garay, Diphenylanthrylene and diphenylfluorene-based segmented conjugated polymer films as fluorescent chemosensors for nitroaromatics in aqueous solution, Sens. Actuators B 203 (2014) 612-620, https://doi.org/10.1016/j.snb.2014.07.032.

[33] R. Garay, A. Schvval, M. Almassio, P. Del Rosso, M. Romagnoli, R. Montani, Fluorescent Amorphous Distyrylnaphthalene-Based Polymers: Synthesis, Characterization and Thin-Film Nanomolar Sensing of Nitroaromatics in Water, Polymers 10 (2018) 1366, https://doi.org/10.1016/j.polymer.2017.02.069.

[34] F. Neese, Software update: the ORCA program system, version 4.0. WIREs Comput, Mol. Sci. (2017) e1327, https://doi.org/10.1002/wcms.1327.

[35] A.R. Allouche, Gabedit - A graphical user interface for computational chemistry softwares, J. Comp. Chem. 32 (2011) 174-182, https://doi.org/10.1002/jcc. 21600.

[36] S. Grimme, J.G. Brandenburg, C. Bannwarth, A. Hansen, Consistent structures and interactions by density functional theory with small atomic orbital basis sets, J. Chem. Phys. 143 (2015) 054107, , https://doi.org/10.1063/1.4927476.

[37] V. Barone, M. Cossi, Quantum calculation of molecular energies and energy gradients in solution by a conductor solvent model, J. Phys. Chem. A 102 (1998) 1995, https://doi.org/10.1021/jp9716997.

[38] T. Ukai, H. Kawazura, Y. Ishii, J.J. Bonnet, J.A. Ibers, Chemistry of dibenzylideneacetone-palladium (0) complexes: I. Novel tris(dibenzylideneacetone)dipalladium (solvent) complexes and their reactions with quinines, J. Organomet. Chem. 65 (1974) 253-266, https://doi.org/10.1016/S0022-328X(00)91277-4.

[39] P.G. Del Rosso, M.F. Almassio, P. Aramendia, S.S. Antollini, R.O. Garay, Poly [(2,2,5,2-tetramethoxy-p-terphenyl-5,5-ylene)propylene]: Synthesis and physical properties of a novel amorphous regularly segmented conjugated polymer, European Polym. J. 43 (2007) 2584-2593, https://doi.org/10.1016/j.eurpolymj. 2007.03.025.

[40] H. Chaumeil, C. Le Drian, A. Defoin, Efficient synthesis of substituted terphenyls by Suzuki coupling reaction, Synthesis 06 (2002) 0757-0760, https://doi.org/10. 1055/s-2002-25773.

[41] Z.E. Lampert, C.L. Reynolds Jr., J.M. Papanikolas, M.O. Aboelfotoh, Controlling morphology and chain aggregation in semiconducting conjugated polymers: The role of solvent on optical gain in MEH-PPV, J. Phys. Chem. B 116 (2012) 12835-12841, https://doi.org/10.1021/jp304199u.
[42] F. Bencheikh, D. Duché, C.M. Ruiz, J.J. Simon, L. Escoubas, Study of Optical Properties and Molecular Aggregation of Conjugated Low Band Gap Copolymers: PTB7 and PTB7-Th, J. Phys. Chem. C 119 (2015) 24643-24648, https://doi.org/10. 1021/acs.jpcc.5b07803.

[43] F.C. Spano, Modeling disorder in polymer aggregates: The optical spectroscopy of regioregular poly (3-hexylthiophene) thin films, J. Chem. Phys. 122 (2005) 234701, , https://doi.org/10.1063/1.1914768.

[44] J. Pommerehne, H. Vestweber, W. Guss, R.F. Mahrt, H. Bässler, M. Porsch, J. Daub, Efficient two layer leds on a polymer blend basis, Adv. Mater. 7 (1995) 551-554, https://doi.org/10.1002/adma.19950070608.

[45] V. Bhalla, A. Gupta, M. Kumar, D.S. Rao, S.K. Prasad, Self-assembled pentacenequinone derivative for trace detection of picric acid, ACS Appl Mater Interfaces. 5 (2013) 672-679, https://doi.org/10.1021/am302132h.

[46] T. Caron, E. Pasquinet, A. van der Lee, R.B. Pansu, V. Rouessac, S. Clavaguera, M. Bouhadid, F. Serein-Spirau, J.P. Lère-Porte, P. Montméat, Efficient Sensing of Explosives by Using Fluorescent Nonporous Films of Oligophenyleneethynylene Derivatives Thanks to Optimal Structure Orientation and Exciton Migration, Chem. Eur. J. 20 (46) (2014) 15069-15076, https://doi.org/10.1002/chem.201402271.

[47] S.D. Kim, J.M. Torkelson, Nanoscale confinement and temperature effects on associative polymers in thin films: Fluorescence study of a telechelic, pyrene-labeled poly (dimethylsiloxane), Macromolecules 35 (2002) 5943-5952, https://doi.org/ $10.1021 / \mathrm{ma0200322.}$

[48] A. El Ouakili, G. Vignaud, E. Balnois, J.F. Bardeau, Y. Grohens, Multiple glass transition temperatures of polymer thin films as probed by multi-wavelength ellipsometry, Thin Solid Films 519 (6) (2011) 2031-2036, https://doi.org/10.1016/j tsf.2010.10.015.

[49] W. Xia, S. Keten, Size-dependent mechanical behavior of free-standing glassy polymer thin films, J. Mater. Res. 30 (2015) 36-45, https://doi.org/10.1557/jmr. 2014.289.

[50] B. Flier, M.I. Baier, M.C. Huber, J. Müllen, K. Mecking, S. Zumbusch, A. Wöll, Heterogeneous Diffusion in Thin Polymer-Films as Observed by High-Temperature Single Molecule Fluorescence Microscopy, J. Am. Chem. Soc. 134 (2012) 480-488, https://doi.org/10.1021/ja208581r.

[51] B. Araoz, A. Carattino, D. Täuber, C. Von Borczyskowski, P.F. Aramendia, Influence of the Glass Transition on Rotational Dynamics of Dyes in Thin Polymer Films: Single-Molecule and Ensemble Experiments, J Phys. Chem. A 118 (45) (2014) 10309-10317, https://doi.org/10.1021/jp500272y.

[52] C.M. Hansen, Hansen Solubility Parameters: A Users Handbook, 2nd Ed., CRC Press LLC, Boca Raton, FL, 2007.

[53] E. Stefanis, C. Panayiotou, Prediction of Hansen solubility parameters with a new group-contribution method, Int. J. Thermophys. 29 (2008) 568-585, https://doi. org/10.1007/s10765-008-0415-z.

[54] E. Stefanis, C. Panayiotou, A new expanded solubility parameter approach, Int. J. Pharm. 426 (2012) 29-43, https://doi.org/10.1016/j.ijpharm.2012.01.001. 